\title{
Dynamic simulation of liquid molecular nanoclusters. Structure, stability and quantification of internal (pseudo)symmetries
}

\author{
Angelo Gavezzotti*,a and Leonardo Lo Presti ${ }^{\mathrm{a}, \mathrm{b}, \mathrm{c}}$ \\ Received (in XXX, XXX) Xth XXXXXXXXX 200X, Accepted Xth XXXXXXXXX 200X \\ ${ }_{5}$ First published on the web Xth $X X X X X X X X X 200 X$ \\ DOI:
}

The atom-atom intermolecular force field AA-CLP is applied to the Molecular Dynamics simulation of liquid nanoclusters of benzene, chloroform, methanol and pyridine. Bulk liquids are also simulated for validation and comparison with experimental data. The applied software has

10 been produced de novo to deal with the unusual analytical form of the intermolecular potential, and includes some novel features for control of net rotational momenta in isolated systems. The nanoclusters have been studied as a function of size (150-1000 molecules) in what concerns cohesion energies, rotational correlation, self-diffusion coefficients, and evaporation rates. Internal structure has been studied with traditional radial distribution functions, plus diagrams of

15 the distribution of intermolecular vectors for flat compounds. In addition, a new algorithm for the quantification of pseudo- or near-symmetries between molecules in aggregates of any structure has been developed and tested, with reference to inversion, mirror plane and rotation axes symmetries proper of organic crystals, with possible import on the investigation of crystallization processes. The results confirm the reliability of the AA-CLP formulation for Molecular Dynamics 20 simulation and throw some light on relationships between cluster and bulk properties. The computer codes are available for open-source download to ensure full reproducibility of all results.

\section{Introduction}

In parallel with an ever growing availability of microscopic ${ }_{25}$ analysis techniques, there is presently an ever growing interest in chemical objects of nanometer dimensions, both on the theoretical and applicative side. These objects have been around in the literature for a long time, since pioneering studies ${ }^{1}$ that used supersonic flow experiments to generate liquid- and crystal-like ${ }_{30}$ molecular nanoparticles, characterizing them by diffraction techniques and molecular dynamics simulation. A recent overview $^{2}$ proves that the subject is still hot today, and although most studies refer to monoatomic gases or, typically, to water, ${ }^{3}$ the experimental and theoretical apparatus is now up to the task of ${ }_{35}$ investigating the nucleation of larger molecules.

* To whom correspondence should be addressed: angelo.gavezzotti@unimi.it

a Department of Chemistry, University of Milano, via Golgi 19, 4020133 Milano, Italy

${ }^{b}$ Istituto di Scienze e Tecnologie Molecolari, Italian CNR,

Via Golgi 19 I-20133 Milano (Italy)

c Centre for Materials Crystallography, Arhus University, Langelandsgade 140, DK-8000 Arhus C. (Denmark)

45

$\dagger$ Electronic Supplementary Information (ESI) available: Force field parameters. Simulation outcomes: supplementary Figures. See DOI:
50

Nanocluster science is an essential part of the development of functional nanoparticles, mostly made of salts or metals, but also organic molecular aggregates is important in many sectors of applied chemistry and physics as, to quote just an example, 55 atmospheric pollution. ${ }^{4}$

Static optimization of the structure of molecular clusters has been pursued by quantum chemical methods. ${ }^{5}$ Such studies involve the determination of temperature-less, static energy minima that may have little to do with the actual behaviour of ${ }_{60}$ these aggregates in real thermal conditions. Molecular dynamics (MD) and Monte Carlo (MC) are by now mature computational techniques of wide applicability. In the cluster arena most frequent applications are to model systems, like the Lennard-Jones sphere, ${ }^{6}$ which allows large particle numbers ${ }_{65}$ but lacks chemical significance, or, again, to the vapor-liquid interface of water droplets, ${ }^{7}$

Rather than in model systems, we are interested in the aggregation properties of polyatomic organic molecules. Currently available MC-MD software include an early but 70 seminal item, GROMOS, ${ }^{8}$ recently evolved into GROMACS. ${ }^{9}$ However, these packages are mainly oriented to the simulation of biological systems and their setup is seldom adaptable to dealing with crystal symmetry or isolated clusters. Besides, they can only be used with the analytical form of the embedded 75 force field. One aim of the present contribution is a validation in MD simulation of the AA-CLP force field, ${ }^{10,11}$ an atom-atom recipe mainly developed for molecular crystals and liquids, up to now only applied in static or Monte Carlo simulation. 
On these premises we present here a suite of Molecular Dynamics programs developed ex novo to treat molecular clusters of up to 1000 molecules of up to 20 atoms, living in a common PC environment, where the code can be easily 5 modified to deal with new situations emerging on the fly, and significant results can be obtained in reasonable solar times without the use of supercomputing resources. Being cheap in the above sense allows a flexible investigation of many different compounds, temperatures and cluster sizes in 10 unforeseen conditions. The formulation uses standard MD algebra but includes some new features specific to isolated clusters, like algorithms for disposal of net overall translational and rotational momentum, or for dealing with evaporation by detachment of boundary molecules from the cluster surface.

15 Molecular clusters are interesting also as precursors of crystal nucleation and growth mechanisms. Therefore, a new algorithm for the quantification of molecular symmetries in systems of any structure between fully liquid and fully crystalline has been developed, with specific reference to symmetries of organic 20 crystals, namely inversion, twofold rotation or screw axes and mirror or glide planes, plus pure translation.

Chemically very different test-case liquids, spanning apolar aromatics (benzene), strongly protic (methanol) and clorurated (chloroform) hydrocarbons, and aromatic bases (pyridine), 25 have been considered. The results prove the reliability of the AA-CLP intermolecular potential scheme in molecular dynamic applications, yielding on the way a number of valuable insights into the structure and evolution of small liquid clusters as a function of size, chemical composition and temperature, 30 also in comparison with bulk liquid properties.

\section{Computational methods}

\section{Intermolecular force field: the AA-CLP scheme}

35 The AA-CLP (Atom-Atom Coulomb-London-Pauli) intermolecular potential ${ }^{10,11}$ is an atom-centered empirical scheme that consists of a Coulombic term over atomic pointcharge parameters, plus terms in inverse powers of interatomic distance $R$ to represent polarization, dispersion and repulsion 40 energies. For the interaction between an $i-j$ atom pair the potential reads: ${ }^{10}$

$$
\begin{aligned}
& E(i, j)=\frac{q_{i} \cdot q_{j}}{R}-\frac{A}{R^{4}}-\frac{B}{R^{6}}+\frac{C}{R^{12}} \\
& \quad=E(\text { Coul })+E(\text { Pol })+E(\text { Dis })+E(\text { Rep })
\end{aligned}
$$

45

Charge parameters $q$ come from Mulliken population analysis on a molecular orbital scheme. $A, B$ and $C$ factors are calculated from basic properties like local charge, ionization potential and polarizability for each atom pair in each molecule. More detail ${ }_{50}$ can be found in the Literature ${ }^{12-16}$ and in the documentation available on line. ${ }^{17}$ This scheme has been optimized to reproduce the properties of a hundreds of organic compounds in the liquid or crystalline state and owes its success to exhaustive parameter fitting. Rather than being set for pairs of 55 atom types, as in most potential schemes, the interaction coefficients are molecule-dependent, which means that the potential cannot be adapted to other available MD packages. For this reason, capital among others, a new molecular dynamics code had to be developed, as described below.

60

\section{Monte Carlo (MC)}

The MC procedure, described ${ }^{11}$ and tested ${ }^{11,18}$ previously, considers any given molecule as either fully rigid (e.g. naphthalene) or partitioned into rigid fragments (like the two

${ }_{65}$ rings in biphenyl or the ring and the $\mathrm{OH}$ group in phenol) connected by rotatable but un-stretchable bonds. The intramolecular force field includes only bond bending for $\mathrm{C}-$ $\mathrm{O}-\mathrm{H}$ fragments and torsional potentials over rotatable bonds. NPT-MC (constant number of particles, pressure and 70 temperature) with periodic boundary conditions is used for liquids and crystalline states.

\section{Molecular Dynamics (MD)}

A description of the essential features of the newly developed $75 \mathrm{MD}$ procedure for clusters follows. More detail can be found in the deposited on line material. ${ }^{19}$

The only structural input to the MD modules is a set of Cartesian atomic position coordinates for the computational box. NPT-MD with periodic boundary conditions of 26 80 replicas of the cubic reference box, with virial pressure control over center-of-mass degrees of freedom, ${ }^{20}$ is applied to simulate bulk liquids. Cluster simulations are by definition carried out in vacuo. The total intermolecular configurational energy is calculated over all atom-atom pairs in all molecule85 molecule pairs below a cutoff distance between centers of mass, usually $18-20 \AA$. In this way the summations run always between neutral charge groups and there is no need of range control in Coulomb terms. Zero-step atomic velocities are assigned by an approximate Maxwellian distribution according 90 to $^{9}$

$$
V=\sqrt{\frac{k_{B} T}{M}}\left[\sum_{i=1}^{12} R_{n i}-6\right]
$$

95 where $k_{B}$ is the Boltzmann constant, $T$ ' is the set temperature, $M$ is the atomic mass, and $R_{n i}$ is a random number between 0 and 1. To reduce translational or rotational biases in small isolated systems the components of any velocity vector $V$ are further randomized as follows:

$$
\begin{gathered}
V=a V_{x}+b V_{y}+c V_{z} \\
b^{2}+c^{2}=1-a^{2} \\
b^{2}=a^{\prime} \cdot\left(1-a^{2}\right) \\
c^{2}=\left(1-a^{\prime}\right) \cdot\left(1-a^{2}\right)
\end{gathered}
$$

with $a$ and $a^{\prime}$ random numbers between 0 and 1. Each component is assigned a plus or minus sign according to a random number being grater or smaller than 0.5 .

Temperature has the standard equipartition expression and is 110 constrained by rescaling all atomic velocities by the ratio between $T$ (set) and $T$ (current) usually every 500-1000 MD steps. In isolated clusters "temperature" is better regarded as just a measure of dynamic freedom with only limited reference to the corresponding thermodynamic quantity, and no pressure 115 control applies.

The trajectory is integrated by a standard leap-frog algorithm 
with time $(t)$, velocity $(V)$, position $(r)$, mass $(M)$ and force $(F):^{9}$

$$
\begin{aligned}
& V(t+1 / 2 \Delta t)=V(t-1 / 2 \Delta t)+\frac{\Delta t}{M} F(t) \\
& r(t+\Delta t)=r(t)+\Delta t \cdot V(t+1 / 2 \Delta t)
\end{aligned}
$$

The intramolecular potentials and forces are taken as such from the GROMOS $96^{20}$ formulation:

$$
\begin{aligned}
& E(\text { str })=1 / 2 \cdot k_{\text {str }}\left(R-R^{0}\right)^{2} \\
& E(\text { bend })=1 / 2 \cdot k_{\text {bend }}\left(\cos \vartheta-\cos \vartheta^{0}\right)^{2} \\
& E(\text { tors })=k_{\text {tors }}[1+f \cdot \cos (m \varphi)]
\end{aligned}
$$

where $R$ is a bond distance, $\vartheta$ is a bond angle, $\varphi$ is a (proper or improper) torsion angle and the $k^{\prime}$ s are parametric force 15 constants. $f$ is a phase factor equal to \pm 1 , and $m$ is an integer equal to 1,2 or 3 . The applied force field includes one stretching potential for each bond, one bending potential for each bond triad, and one torsion potential along each chemical bond. Improper dihedrals help preserving planar centers (e.g. $20 s p^{2} \mathrm{C}$-atoms in aromatic rings). The force constants are assigned values from the literature ${ }^{21}$ or (especially for torsions) from ab initio calibration; for nearly rigid systems, the choice of force constants is only moderately influential as highfrequency internal vibrations are not relevant to the problem at 25 hand, namely intermolecular structure and phase change. Intramolecular potentials serve only as restraints to avoid undue molecular bond and angle distortions. At least for molecules without extensive internal flexibility degrees of freedom, the simple setup performs well with considerable time 30 savings over more complex analytical constraint procedures. All numerical data are collected in ESI-Force field (Section S1 ESI).

Unwanted net momenta. In MD simulations of isolated clusters net translational and rotational momenta may develop.

35 Empirical procedures have been devised to deal with of these adverse factors. The array of centers of mass at step zero is taken as a reference configuration. To dispose of $\mathrm{Tr}$-drift, all coordinates are reset to the origin of the current center of mass (c.o.m) every 20-50 MD steps. To suppress Rot-momentum a 40 rotation matrix is prepared as the product of three rotation matrices, one around each inertial axis, and an array of possible back-rotations of the whole cluster is explored by varying the three rotation angles from -15 to $+15^{\circ}$ in steps of $3^{\circ}$. The backrotation actually performed is the one for which the sum of the

${ }_{45}$ distances between current centers of mass and the reference configuration at zero simulation time is minimum. These corrections avoid complex algebra and affect the whole set of atomic coordinates in a rigid manner without discontinuities in the trajectories. Their efficiency has been checked by tests on 50 correlation functions and distributions of centers of mass with and without the corrections.

Evaporation. In MD runs on isolated clusters some surface molecules may drift away in the simulation analog of evaporation. Two procedures are introduced to deal with this ${ }_{55}$ problem. In the first, if the c.o.m. of a molecule is farther from the overall c.o.m. of the cluster by more than an assigned threshold, $\mathrm{R}_{\mathrm{ev}}$, the c.o.m. vector is reduced by a factor $0<\mathrm{F}_{\mathrm{ev}}$ $<1$ so that the whole molecule is "tethered" back by a vector
$1-\mathrm{F}_{\mathrm{ev}}$. Using $\mathrm{F}_{\mathrm{ev}} \approx 0.9$ and $\mathrm{R}_{\mathrm{ev}} \approx 1.5$ times the cluster radius a ${ }_{60}$ few molecules are thus kept in orbit, being then excluded from the estimation of rms displacements. In the second procedure when a c.o.m vector exceeds the diameter of the cluster the molecule is simply deleted from the simulation. This applies for larger clusters where deletion of a few molecules is not ${ }_{65}$ influential, or when evaporation rates are estimated.

Trajectory analysis. Periodic checks are carried out to make sure that molecular distortions do not exceed a limit of a few $R T$ units. The intermolecular structure of the sample is described by radial distribution functions (RDF):

70

$$
g\left(R_{k}\right)=\frac{N\left(R_{k}\right)}{4 \pi R_{k}^{2} \mathrm{~d} R} \cdot\left(\frac{N}{V}\right)^{-1}
$$

where $N\left(R_{k}\right)$ is the number of distances in the $k$-th distance bin and is divided by the volume of the bin of thickness $\mathrm{d} R$ (usually $750.1-0.2 \AA)$. The term $(N / V)^{-1}$ normalizes the RDF to the number density. The volume of an isolated cluster is approximated by the sum of molecular volumes divided by a packing coefficient of 0.6 .

Rotational correlation is estimated by the following 80 expression:

$$
C(\mathbf{u})=100 \cdot\langle\mathbf{u}(t) \cdot \mathbf{u}(0)\rangle
$$

where $\mathbf{u}$ is a unit vector along any chosen molecular axis at time $t$ with respect to a chosen reference. The brackets denote the 85 average of dot products over the whole simulation sample. The rotational correlation time is estimated as the time for $C(\mathbf{u})$ to decay from 100 to about 20, and should be of the order of 5-20 ps for organic liquids. The translational diffusion coefficient $D$ is given by the Einstein relation:

$$
D=\frac{1}{6 \Delta t} \cdot\langle\mathbf{d}(t)-\mathbf{d}(0)\rangle
$$

where $\mathbf{d}(t)$ is the placement vector of the center of mass at time $t$ and $\mathbf{d}(0)$ is the chosen reference. $D$ is calculated as $1 / 6$ of the 95 slope of the quadratic displacement versus time and should be of the order of $10^{-8} \mathrm{~m}^{2} \mathrm{~s}^{-1}$ ( or $\AA^{2} \mathrm{ps}^{-1}$ ).

Reproducibility. Fortran source modules, sample input and output data with documentation of the computational scheme 100 are available for download from www.angelogavezzotti.it, CLP-dyn link. Together with deposited force field data (Section S1 ESI) this ensures complete reproducibility of all calculations.

\section{${ }_{105}$ Generation of starting aggregates}

Molecular models of standard geometry (see ESI,Section S1) are used to prepare approximate starting computational boxes with molecules in random orientation. A sequence of energy minimization and NPT-MC runs produces equilibrated models 110 of the liquids. The resulting final computational boxes are input as such to the NPT-MD simulations to check the correct reproduction of bulk liquid properties. Then, roughly globular, isolated nanoclusters (droplets) of variable size are prepared by deleting outer molecules. The clusters are input to starting MD 115 runs of $60-100$ ps followed by production runs of $100 \mathrm{ps}$, with 
a timestep of 2 fs. Such lengths are enough to characterize the main dynamic features, rotational correlation and selfdiffusion. Longer runs are used to explore evaporation. C.o.m. and atom-atom radial distribution functions, eq. (6), are 5 calculated on the final MD frames. C.o.m. diffusion coefficients, eq. (8), and rotational correlation functions, eq. (7), are calculated from the resulting MD trajectories.

\section{Quantification of symmetries in a molecular array}

${ }_{10}$ Molecular clusters are interesting in themselves, but also in the perspective of a possible transition to crystalline structure along the nucleation and growth processes. Through phase changes, a crucial parameter is therefore the degree of crystallinity of the system. A simple but efficient way to obtain

15 a quantitative measure of this parameter is described in the following.

In any pair of mlecules of a static computer model of an ordered molecular crystal or of a molecular liquid composed of rigid molecules, the pair members are related by an isometry, 20 i.e. a distance-preserving transformation. In a perfect crystal the molecules are also related by perfect symmetry, for organic crystals by translation $T$, inversion $I$, twofold screw axis $S$ and glide mirror plane $G$; less common are twofold axis $A$ and mirror plane $M$. In what follows the translations associated 25 with $\mathrm{G}$ and $\mathrm{S}$ operators can be neglected and their symmetry reduces to $\mathrm{M}$ or $\mathrm{A}$ respectively, making the analysis much easier.

Consider an ensemble of $N_{\text {mol }}$ molecules each of $N_{\text {at }}$ atoms, in which molecule $i$ is described by the set of atomic coordinates $30 w_{\mathrm{i}}{ }^{\circ}[\mathrm{x}, \mathrm{y}, \mathrm{z}]$ with center of mass $w_{\mathrm{im}}{ }^{\circ}$, in any external reference system. Firstly, all molecules are set in the reference system of their center of mass, $w_{\mathrm{i}}^{\prime}=w_{\mathrm{i}}{ }^{\circ}-w_{\mathrm{im}}{ }^{\circ}$. If the $i-j$ pair is related by a perfect symmetry, the two sets of coordinates $w_{\mathrm{i}}^{\prime}$ and $w_{\mathrm{j}}^{\prime}$ are identical, except for some combination of sign inversions (first 35 column of Table 1). In general for rigid or flexible molecules in any environment, a numerical index of deviation from symmetry can be constructed as follows. The overlap between the two sets of coordinates is maximized by transformation to the common inertial reference system. The six possible sums 40 over the resulting final coordinates of the two partners, from $\Sigma_{(1)}$ to $\Sigma_{(6)}$, are then computed:

45

$$
\begin{aligned}
& \Sigma_{(1)}=\frac{1}{N_{a t}} \cdot \sum_{i j}\left|x_{i}+x_{j}\right| \\
& \Sigma_{(2)}=\frac{1}{N_{a t}} \cdot \sum_{i j}\left|x_{i}-x_{j}\right| \\
& \Sigma_{(3)}=\frac{1}{N_{a t}} \cdot \sum_{i j}\left|y_{i}+y_{j}\right| \\
& \Sigma_{(4)}=\frac{1}{N_{a t}} \cdot \sum_{i j}\left|y_{i}-y_{j}\right| \\
& \Sigma_{(5)}=\frac{1}{N_{a t}} \cdot \sum_{i j}\left|z_{i}+z_{j}\right| \\
& \Sigma_{(6)}=\frac{1}{N_{a t}} \cdot \sum_{i j}\left|z_{i}-z_{j}\right|
\end{aligned}
$$

${ }_{50}$ where each sum runs over all $N_{\text {at }}$ pairs of corresponding atoms in the two molecules. If the molecule has internal point-group symmetry, the six sums are evaluated for each equivalent permutation of atom numberings generated by the point-group symmetry, and the lowest values are taken.

55 If the molecular pair is exactly symmetric three of these sums are exactly zero (Table 1). For any molecular pair not necessarily endowed with symmetry, the six sums are ranked in ascending order and it is assumed that the three lowest ones are the best approach to a possible symmetry relationship; their ${ }_{60}$ sum can be called an index of symmetry breaking, $g_{\mathrm{ij}}$, in units of $\AA$ per atom (eq. 10). The average of $g_{\mathrm{ij}}$ 's over all pairs in the entire molecular array is the global asymmetry index of the array, $G$ (eq. 11), representing an inverse order parameter:

65

$$
\begin{aligned}
& g_{i j}=\sum_{i=1}^{3} \min \left(\Sigma_{i}\right) \\
& G=\frac{1}{N_{\text {pairs }}} \cdot \sum_{i<j} g_{i j}
\end{aligned}
$$

\begin{tabular}{|c|c|c|c|c|c|c|}
\hline $\begin{array}{l}\text { symmetry } \\
\text { relationship }\end{array}$ & \multicolumn{6}{|c|}{$\Sigma_{(i)}$} \\
\hline (i) & (1) & (2) & (3) & (4) & (5) & (6) \\
\hline$x, y, z$ & any & 0 & any & 0 & any & 0 \\
\hline$-x, y, z \quad G, M$ & 0 & any & any & 0 & any & 0 \\
\hline$x,-y, z$ & any & 0 & 0 & any & any & 0 \\
\hline$x, y,-z \quad G, M$ & any & 0 & any & 0 & 0 & any \\
\hline$-x,-y, z \quad S, A$ & 0 & any & 0 & any & any & 0 \\
\hline$-x, y,-z \quad S, A$ & 0 & any & any & 0 & 0 & any \\
\hline$x,-y,-z \quad S, A$ & any & 0 & 0 & any & 0 & any \\
\hline$-x,-y,-z \quad I$ & 0 & any & 0 & any & 0 & any \\
\hline
\end{tabular}

where $N_{\text {pairs }}=N_{\text {mol }}\left(N_{\text {mol }}-1\right) / 2$. More revealing than just the global index $\mathrm{G}$ can be the distribution of $g_{\mathrm{ij}}$.'s over the entire 70 sample: a peak near $N\left(\mathrm{~g}_{\mathrm{ij}}\right)=0$ indicates approximate crystalline symmetry while a flat profile denotes an aggregate close to the liquid state.

Table 1. Values of the six sums for perfect symmetries. $T=$ translation, ${ }_{75} G, M=$ glide or mirror plane, $S, A=$ twofold screw or axis, $I=$ inversion.

\section{Results}

\section{Bulk liquids vs. clusters}

Cohesive energies and densities. For bulk liquids the results of the NPT-MC treatment (Table 2) reproduce the experimental conditions, as expected from previous calibration. ${ }^{10,11}$ The NPTMD results are also in good correspondence with experiment, in a preliminary validation of the whole setup and of the potential energy schemes for molecular dynamics application The ${ }_{85}$ comparison between cohesive energies and experimental vaporization enthalpies is only qualitative, since no account is taken of possible changes in intramolecular energies between liquid and vapor. The agreement between calculated and observed liquid densities is satisfactory, confirming that the potentials place ${ }_{90}$ their minima at the correct position, and parallels the acceptable reproduction of intermolecular energetics. Differences between $\mathrm{MC}$ and MD results may come from differences in box dimensions and treatment of intermolecular flexibility. MD performs even better than MC although smaller sample boxes are used.

${ }_{95}$ Intermolecular orientation angles. For flat molecules like benzene or pyridine, one would think intuitively that ring stacking might be favored in condensed aggregates. This is not the case in the crystal structures of both compounds, where $\mathrm{T}$-shaped arrangement predominates. This feature can be probed by calculating the distribution of angles between the normal to the 
ring in molecular pairs, $\delta$. Results for the angles between ring normals in benzene are shown in Figure 1: the distribution shows a decrease in frequency of higher angles at short distances, as expected. By contrast, the distribution in a crystalline aggregate reveals sharp peaks for the symmetry-related molecular orientations (Figure S1 ESI). The distribution of angles between molecular $\mathrm{C}-\mathrm{H}$ vectors in chloroform clusters is on the contrary perfectly uniform in distance (ESI, Figure S2).

Quantitative treatment of orientation probability densities in bulk ${ }_{10}$ systems $^{22-24}$ could be given for a more detailed discussion of the matter; this would be however outside the scope of the present work. We merely point out that the distribution of inter ring angles in liquid benzene clusters is identical to that of the bulk liquid (ESI, Figure S3).

Table 2. Results of NPT-periodic boundary MC and MD simulation for bulk liquids.

\begin{tabular}{|c|c|c|c|c|c|c|}
\hline \multirow[b]{2}{*}{ Substance } & \multirow[b]{2}{*}{ Method } & \multicolumn{2}{|c|}{ Energies $/ \mathrm{kJ} \cdot \mathrm{mol}^{-1}$} & \multicolumn{2}{|c|}{ Density $/ \mathrm{g} \cdot \mathrm{cm}^{-3}$} & \multirow{2}{*}{$\begin{array}{l}\mathrm{n} \\
\text { molecules } \\
\mathrm{c}\end{array}$} \\
\hline & & $\mathrm{CE}^{\mathrm{a}}$ & $\operatorname{exptl} \Delta_{\text {vap }} H^{\mathrm{b}}$ & Calc & Exptl & \\
\hline benzene & $\begin{array}{l}\text { MC } \\
\text { MD }\end{array}$ & $\begin{array}{l}31.6(2) \\
32.5(5)\end{array}$ & 34 & $\begin{array}{l}0.897(2) \\
0.917(9)\end{array}$ & 0.87 & $\begin{array}{l}686 \\
250\end{array}$ \\
\hline chloroform & $\begin{array}{l}\mathrm{MC} \\
\mathrm{MD}\end{array}$ & $\begin{array}{l}26.8(1) \\
26.4(3)\end{array}$ & 31 & $\begin{array}{l}1.410(2) \\
1.451(10)\end{array}$ & 1.47 & $\begin{array}{l}1458 \\
432\end{array}$ \\
\hline methanol & $\begin{array}{l}\text { MC } \\
\text { MD }\end{array}$ & $\begin{array}{l}37.8(1) \\
42.6(5)\end{array}$ & 37 & $\begin{array}{l}0.770(1) \\
0.799(10)\end{array}$ & 0.79 & $\begin{array}{l}1458 \\
432\end{array}$ \\
\hline pyridine & $\begin{array}{l}\mathrm{MC} \\
\mathrm{MD}\end{array}$ & $\begin{array}{l}39.2(2) \\
38.9(7)\end{array}$ & 40 & $\begin{array}{l}1.047(5) \\
1.040(13)\end{array}$ & 0.982 & $\begin{array}{l}432 \\
250\end{array}$ \\
\hline
\end{tabular}

${ }^{a}$ Cohesive intermolecular energy. Rmsd in parentheses from the average in production runs.

${ }_{20}$ brom https://webbook.nist.gov/chemistry/.

cNumber of molecules in central box.

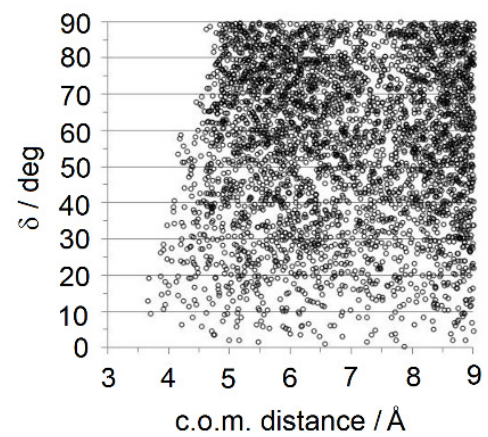

Figure 1. The distribution of inter-ring angles, $\delta$, computed as the 25 angle between ring normals, in the final frame of the MD simulation of a 475-molecule benzene cluster. At short c.o.m. distances small angles predominate, corresponding to approximate stacking. The distribution in the final frame of the bulk liquid simulation is nearly identical.

30

Nanocluster properties. In the MD runs for clusters the intramolecular force fields keep the molecules undistorted, with torsion angles oscillations $<10^{\circ}$, rms bond stretchings of $0.03 \AA$ and rms bond angle bendings of $3.5^{\circ}$, and corresponding 35 strain energies of 0.6 to $1.5 \mathrm{~kJ} \cdot \mathrm{mol}^{-1}$ per intramolecular degree of freedom. The few evaporating surface molecules are taken care of by the tethering or deletion strategies. All considered, MD production runs for nanoclusters are stable and robust.

Graphs for the correlation functions are given in Figure 2. ${ }_{40}$ Calculated dynamic properties in Table 3 generally show a reasonable agreement with experimental data and other simulation results ${ }^{25-28}$ (which are also not free from oscillations, see footnotes to Table 3) and nicely reflect the different strength of intermolecular interactions, from weakly 45 bound benzene or chloroform to tighter binding in pyridine and even more in the hydrogen-bonded alcohol. Self-diffusion coefficients and rotational correlation times in clusters are both overestimated with respect to bulk liquid, presumably due to an imbalance in boundless systems where diffusion (expansion) 50 takes the best part of kinetic energy. As expected, the benzene rotational correlation time is higher for the rotation axis with the higher moment of inertia (perpendicular to the ring).

The results in Table 3 further confirm the reliability of the computational scheme. A somewhat unexpected, new result is 55 that rotational correlation times are identical in the bulk liquid and in nanoclusters made of 400-1000 molecules (see also Figure S4 ESI).

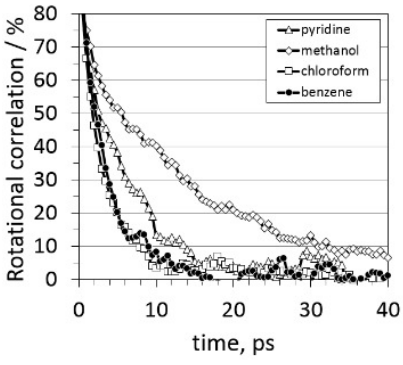

(a)

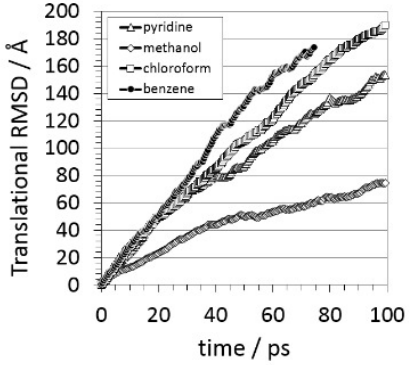

(b)
Figure 2. Time evolution of (a) rotational correlation function and (b) 60 translational root mean squared c.o.m. displacement, as obtained from the $100 \mathrm{ps}$ MD production runs for liquid clusters (see Table 3).

Table 3. Correlation results from MD. Self-diffusion coefficients from the slope of the rmsd vs. time graphs in Figure 2 from cluster calculations.

\begin{tabular}{|c|c|c|c|c|c|c|}
\hline & \multicolumn{2}{|c|}{ Rotational correlation, $\mathrm{ps}$} & \multicolumn{2}{|c|}{$\begin{array}{l}\text { Self-diffusion } \\
\text { Coefficient } 10^{-9} \mathrm{~m}^{2} \mathrm{~s}^{-1}\end{array}$} & \multicolumn{2}{|c|}{$\mathrm{n}$ (molecules) } \\
\hline & $\mathrm{Calc}^{\mathrm{a}}$ & Exptl & $\mathrm{Calc}^{\mathrm{b}}$ & Exptl & Cluster & bulk \\
\hline $\begin{array}{l}\text { benzene } \\
\text { chloroform }\end{array}$ & $\begin{array}{l}6.7(\|), 4.5(\perp)^{c} \\
4.9\end{array}$ & $4.7(\|), 3.0(\perp)^{\mathrm{c}}$ & $\begin{array}{l}4.2 \\
3.4\end{array}$ & $\begin{array}{l}2.3^{\mathrm{c}} \\
2.3^{\mathrm{d}}\end{array}$ & & \\
\hline methanol & $\begin{array}{l}4.9 \\
20\end{array}$ & $\begin{array}{l}15^{\mathrm{e}}-3 \\
1.5\end{array}$ & 1.4 & $1.3-2.3^{\mathrm{e}}, 2.4^{\mathrm{f}}$ & 958 & 432 \\
\hline pyridine & 9.1 & $3.0^{\mathrm{g}}$ & 2.8 & $1.88^{\mathrm{g}}$ & 432 & 250 \\
\hline
\end{tabular}

${ }_{65}{ }^{\text {a }}$ Same results for clusters or bulk.

${ }^{\mathrm{b}}$ Cluster.

${ }^{\mathrm{c}}$ MD simulation and experiment, ref. 15; $(\|)$ parallel, $(\perp)$ perpendicular to the ring plane normal.

${ }^{\mathrm{d}}$ As quoted in ref. 25 along with MD results.

$70{ }^{\mathrm{e}} \mathrm{MD}$ calculation, ref. 26.

${ }^{\mathrm{f}}$ As quoted in ref. 27, with comparable DFT-MD results.

${ }^{g}$ As quoted in ref. 28, along with similar results from MD calculations.

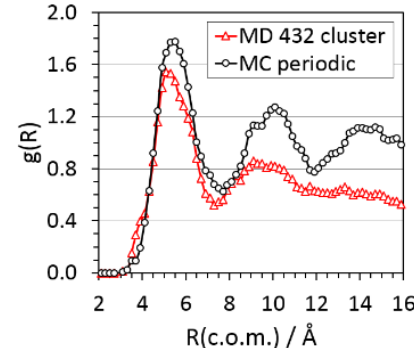

(a)

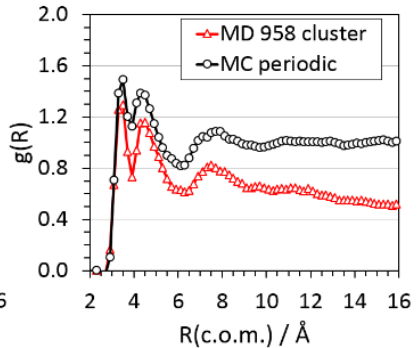

(b)
75 Fig. 3. Center-of-mass radial distribution functions, $g(R)$, for bulk liquid from $\mathrm{MC}$ and for clusters from MD. (a) pyridine; (b) methanol.

Figure 3 shows a comparison of the center-of-mass radial distribution functions from bulk MC simulation and for clusters: 
they are in agreement with the results of accurate literature simulations on bulk liquids as well as with results from periodicbox simulation of bulk liquids. Similar results are obtained also for benzene and chloroform (see also Figure S5 ESI). The MD 5 description of the hydrogen bond in methanol is adequate, with about one bond per molecule and RDF peaks at 1.8, 2.2 and 2.8 for the $\mathrm{O} \cdots \mathrm{H}, \mathrm{H} \cdots \mathrm{H}$ and $\mathrm{O} \cdots \mathrm{O}$ distances, respectively (see Figure S6 ESI). The dynamic simulation correctly reproduces the effect of surface tension in changing a starting cubic slab into a 10 spherical drop (Figure 4). Again remarkably, RDF's from large clusters are identical to those of bulk liquids.

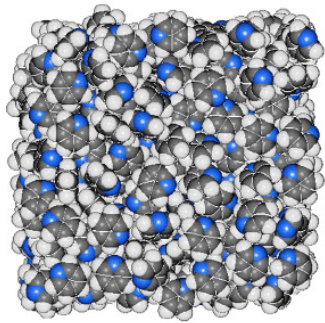

(a)

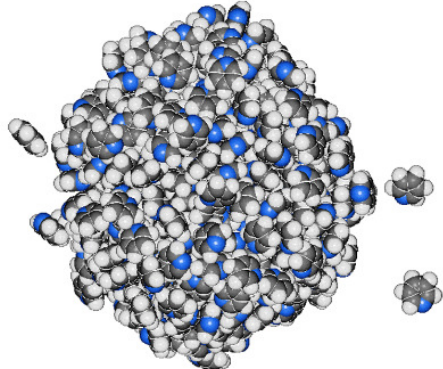

(b)
Figure 4. MD simulation of an isolated cluster of liquid pyridine. (a) Starting cubic slab; (b) final spherical droplet, showing a few incipient 15 evaporated molecules.

\section{Use of symmetry indices $g_{i j}$ and $G$}

During production runs of both MC and MD simulation for all considered liquids, the global $G$-index of eq. (11) is strictly 20 constant. This is a valuable additional signal that the simulation has reached equilibration. Figure 5 shows the distribution of $g_{i j}$ indices in the final frames of the cluster MD simulations. The trace for methanol shows a single peak more or less symmetric around the average, but the trace for pyridine is strongly skewed 25 to the left, indicating that smaller values of $g_{\mathrm{ij}}$ predominate. According to this result the pyridine liquid counts a relatively large number of molecular pairs with some sort of approximate symmetry. Based on molecular shape, one can only guess that this is due to partial stacking of the aromatic rings. According to this

30 result the pyridine liquid counts a relatively large number of molecular pairs with some sort of fractional symmetry, presumably of the translational or inversion type as proper of near bimolecular stacking.

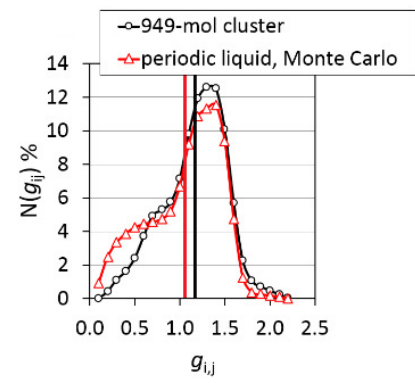

(a)

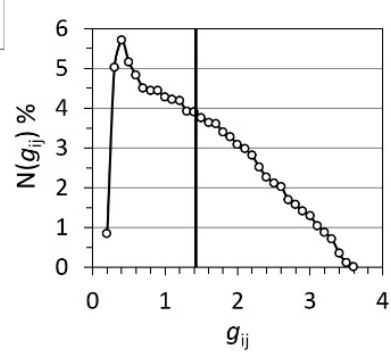

(b)
35 Figure 5. (a) The distribution of $g_{\text {ij }}$ across the final frames of methanol. (b) Same as (a), for pyridine (427-mol cluster). Vertical bars indicate the corresponding average values.
The distribution of $g_{\mathrm{ij}}$ can also be represented as a function of distance between centers of mass: the result for benzene, and even 40 more for pyridine (Figure 6) shows that the closest molecular pairs (which also have smaller interplanar angles, see Figure 1) tend to have a higher degree of symmetry. The same trend is observed in chloroform, but not in methanol. A possible interpretation is that closeness plays a role in inducing symmetry 45 for systems whose aggregation is dictated by shape rather than by stronger interactions like a hydrogen bond. ${ }^{29}$ These indications are by necessity somewhat qualitative. More experience on the use of this novel function is needed before clear-cut structural conclusions can be drawn.

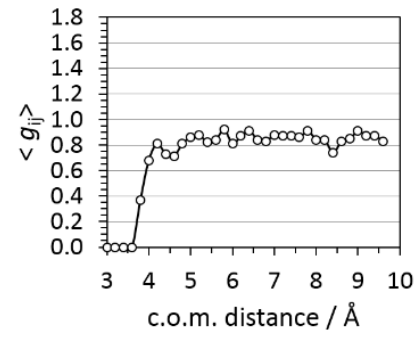

(a)

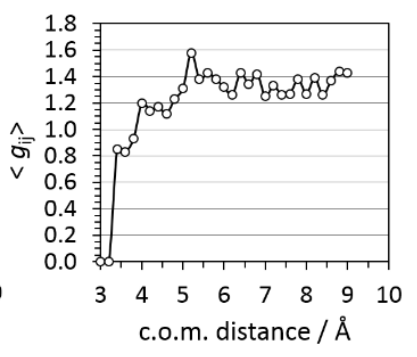

(b)
Fig. 6. Graphs for the average value of $g_{\text {ij }}$ as a function of intermolecular distance between centers of mass. (a) Benzene cluster, 475 molecules at the last step. (b) Pyridine cluster, 427 molecules at the last step. Closer 55 molecular pairs tend to have higher symmetry relationship.

\section{Cluster size effects and evaporation rates}

Simulations have been carried out for chloroform and for benzene with variable cluster sizes. Figure 7 and Table 4 show the results. ${ }_{60}$ For chloroform, smaller clusters have slightly smaller rotational correlation times; as the cluster becomes large enough, the correlation time becomes identical to that of the bulk liquid. Smaller clusters also have smaller cohesive energies and higher rms center-of-mass displacements, as expected. A cluster of ${ }_{65}$ about 1000 molecules is in all these respects quite similar to the bulk liquid. For benzene the results are a bit more erratic, and a 500-molecule cluster is still somewhat far away from the properties of bulk liquid. There seems to be an indication that cluster size is influential for benzene but not for chloroform, but 70 the differences in rotational decay times could also be within the confidence intervals of the corresponding quantities.

Table 4. Results of variable-size cluster simulations for chloroform and benzene .

\begin{tabular}{|c|c|c|c|c|c|c|}
\hline \multirow[b]{2}{*}{$\begin{array}{l}\mathrm{n} \\
\text { (molecules) }\end{array}$} & \multirow[b]{2}{*}{$\begin{array}{l}\text { radius, } \\
\AA\end{array}$} & \multirow[b]{2}{*}{$t(20)^{\mathrm{a}}$} & \multirow[b]{2}{*}{$\operatorname{RMSD}(10)^{\mathrm{b}}$} & \multirow[b]{2}{*}{$\begin{array}{l}E_{\mathrm{coh}^{\mathrm{c}}} \\
/ \mathrm{kJ} \cdot \mathrm{mol}^{-1}\end{array}$} & \multicolumn{2}{|c|}{ Evaporation rate } \\
\hline & & & & & $\mathrm{mol} / \mathrm{ps}$ & $\begin{array}{l}10^{5} \\
\mathrm{~mol} /\left(\mathrm{ps} \AA^{2}\right)\end{array}$ \\
\hline \multicolumn{7}{|l|}{$\mathrm{CHCl}_{3}$} \\
\hline 102 & 13 & 3.2 & 40 & 16 & 0.05 & 2.3 \\
\hline 260 & 19 & 3.7 & 44 & 20 & 0.08 & 1.7 \\
\hline 514 & 24 & 4.5 & 43 & 22 & 0.08 & 1.4 \\
\hline 998 & 31 & 5.2 & 27 & 26 & 0.11 & 0.9 \\
\hline bulk & - & 4.7 & - & 26 & - & - \\
\hline \multicolumn{7}{|l|}{$\mathrm{C}_{6} \mathrm{H}_{6}$} \\
\hline 149 & 17 & 6.1 & 41 & 24 & 0.09 & 2.5 \\
\hline 278 & 21 & 5.7 & 40 & 26 & 0.04 & 0.8 \\
\hline 479 & 26 & 5.1 & 28 & 27 & 0.04 & 0.5 \\
\hline bulk & - & 6.7 & - & 33 & - & - \\
\hline
\end{tabular}

75 a Time (ps) for rotational correlation to decay from 100 to $20 \%$.

${ }^{\mathrm{b}}$ Root-mean-square center-of-mass displacement after $10 \mathrm{ps}$ simulation (starting diffusion; $\AA$ ).

${ }^{\mathrm{c}}$ Cohesive intermolecular energy $(\mathrm{kJ} / \mathrm{mol})$. 
The simulations of evaporation were carried out by deleting the molecules for which the distance between the center of mass and the cluster center of mass exceeds the diameter of the cluster (see 5 "Evaporation" in the Methods Section). Longer simulation times (up to $500 \mathrm{ps}$ ) were carried out in order to observe a sizeable number of evaporated molecules. The absolute evaporation rate, in molecules per picosecond, increases with increasing cluster surface, but the rate normalized to surface decreases due to 10 increasing cohesion in larger clusters. An estimate of the lifetime of a 1000-molecule chloroform cluster or of a $500-$ molecule benzene cluster at room temperature, assuming a constant evaporation rate, is then about $10 \mathrm{~ns}$.

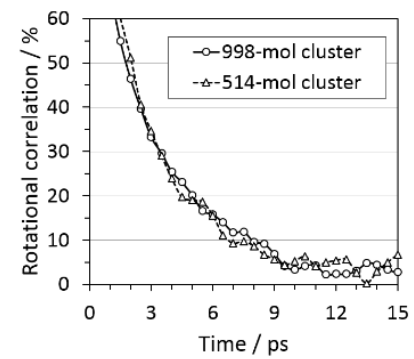

(a)

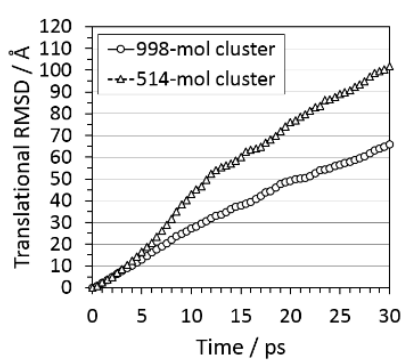

(b)
15 Figure 7. Correlation functions for chloroform vs. simulation time. (a) Rotational coefficient. (b) Translational root mean squared displacement. The results for benzene (see ESI, Figure S4) are quite similar.

\section{Conclusions}

20 This work describes a Molecular Dynamics (MD) formulation, in part using standard algorithms and in part explicitly devised for application to a study of the dynamic properties of isolated liquid nanoclusters, or droplets. Standard stretch-bend-torsion force fields are used to control intramolecular deformation without 25 recourse to comlex constraint algorithms. The software includes simple, time-saving methods for controlling net rotational and translational momenta and for dealing with evaporation in isolated systems. The AA-CLP intermolecular force field is employed, originally parametrized to reproduce the bulk 30 properties of molecular crystals and liquids. The setup is tested against nanoclusters of 150-1000 molecules of benzene, chloroform, methanol and pyridine, analyzing their cohesion energies, rotational correlation, self-diffusion coefficients, evaporation rates and internal structure. A newly developed 35 algorithm is presented for the analysis of hidden or emerging molecule-molecule symmetry relationships, with reference to common space-group symmetry operations like inversion, screw or twofold rotation axes, and mirror or glide planes. On the other hand, this Molecular Dynamics setup includes a number of 40 approximations, especially in the treatment of intramolecular flexibility, of temperature and pressure control, that prevent it from standing up against more refined and widely used MD packages. It should be taken rather as a tool and a help alongside the exploration of experimental occurrences in the realm of 45 nanosized aggregates, not being intended as a device for the delivery of quantitative termophysical quantities by simulation. A more detailed summary of main results follows. (a) Overall, appraisal of the applications confirms the reliability of the AA-CLP formulation for Molecular Dynamics 50 simulations, complementing the already established reliability of the method in static lattice energy calculations and in Monte Carlo simulation.

(b) Simulations on nanoclusters for different chemistry (a hydrocarbon, a chloro- derivative, an azahydrocarbon, and a 55 hydrogen bonded species) reveal the differences in dynamic behavior related to the relative strengths of intermolecular forces. Molecular mobility, in terms of rotational correlation times and self-diffusion coefficients, increases at smaller cluster sizes. Larger, 500- to 1000-molecule clusters are already quite similar 60 to bulk liquids in internal structure (radial distribution functions), and thermochemical and dynamic properties (intermolecular correlation times).

(c) Evaporation rates are estimated by the number of molecules that leave an isolated cluster as a function of time and cluster ${ }_{65}$ surface area. For example, a 1000-molecule nanodroplet of a highly volatile substance like chloroform is predicted to have a

lifetime of about $10 \mathrm{~ns}$ in isolation, while a cluster of hydrogenbonded methanol molecules with similar dimensions is much more persistent.

70 (d) Using the above mentioned symmetry-control algorithm, no trace of persistent symmetric relationships are detected in bulk liquids or in clusters; however, a small but significant increase in symmetric arrangements appears for nearest neighbors of flat molecules like benzene and pyridine. These are presumably 75 shape-driven due to incipient stacking, as demonstrated by the increase of small interplanar angles at short distance between molecular centers. This effect is absent in methanol and chloroform, where shape effects play a minor role. At least in principle, the algorithm shows promise for application to future 80 studies of phase transitions (crystal nucleation and growth).

(e) Our simulations have been conducted for time lengths necessary and sufficient to characterize the main dynamic features of the sample. For bulk liquids, already heavily optimized by preliminary MC treatment, longer duration would 85 have carried no new information. For isolated clusters, except for evaporation studies, simulation for a few hundred picoseconds was more than enough to allow the estimate of correlation times and to characterize structures. All calculations have been carried out in reasonable solar times of the order of a few hours on 90 ordinary PCs without recourse to special computing resources; this approach might help transferring molecular simulation into the usual arsenal of general and experimental chemists, rather than being restricted to a small community of computer experts.

Finally, it is found that Molecular Dynamics supported by the ${ }_{95} \mathrm{AA}-\mathrm{CLP}$ potentials is able to reproduce key macroscopic thermodynamic quantities and to give a good picture of the dynamic behavior of organic liquids and droplets. As such it may provide a viable approach to the molecular-level study of the dynamics of nanoclusters in general, with potential application in 100 non-equilibrium solution chemistry, exploring complex collective phenomena such as supersaturation / supercooling, the early stages of crystal nuclei formation and phase transitions, and 
spinodal decompositions. Such developments will be explored in forthcoming studies.

Acknowledgements. One author (LLP) wishes to thank 5 Università degli Studi di Milano (Department of Chemistry) for

\section{References}

${ }^{1}$ L. S. Bartell, Chem. Revs. 1986, 86, 491-505.

${ }^{2}$ B. E. Wyslouzil and J. Wölk, J. Chem. Phys. 2016, 145, 211702 1,26 .

${ }^{3}$ Y. J. Kim, B. E. Wyslouzil, G. Willemski, J. Wölk and R. Strey, J. Phys. Chem. 2004, 108, 4365-4377.

${ }^{4}$ F. Yu and R. P. Turco, J. Geophys. Res. 2001, 106, 4797-4814.

${ }^{5}$ S.R. Gadre, S. D. Yeole and N. Sahu, Chem. Revs. 2014, 114, 12132-12173.

${ }^{6}$ R. Angelil, J. Diemand, K. K. Tanaka and H. Tanaka, J. Chem. Phys. 2014, 140, 074303.

${ }^{7}$ H. Matsubara, J. Chem. Phys. 2007, 127, 214507

${ }^{8}$ W. F. van Gunsteren and H. J. C. Berendsen, Angew. Chem. Int. Ed. 1990, 29, 992-1023.

${ }^{9}$ See at http://www.gromacs.org

${ }^{10}$ A. Gavezzotti, New J. Chem. 2011, 35, 1360-1368.

${ }^{11}$ A. Gavezzotti, New J. Chem. 2013, 37, 2110-2119.

${ }^{12}$ A. Gavezzotti and L. Lo Presti, Crystal Growth Des. 2016, 16, $2952-2962$.

${ }^{13}$ A. Gavezzotti, V. Colombo and L. Lo Presti, Crystal Growth Des. 2016, 16, 6095-6104.

${ }^{14}$ V. Colombo, L. Lo Presti and A. Gavezzotti, CrystEngComm, 2017,19, 2413-2423.

${ }^{15}$ L. Lo Presti, CrystEngComm, 2018, 20, 5976-5989.

${ }^{16}$ A. Gavezzotti, S. Rizzato and L. Lo Presti, Cryst. Growth Des., 2018, 18, 7219-7227.

${ }^{17}$ A. Gavezzotti, The Coulomb - London - Pauli (CLP) model of intermolecular interaction, CLPdyn, Monte Carlo and Molecular Dynamics modules, Description and user manual,

\section{For Table of Contents use only}

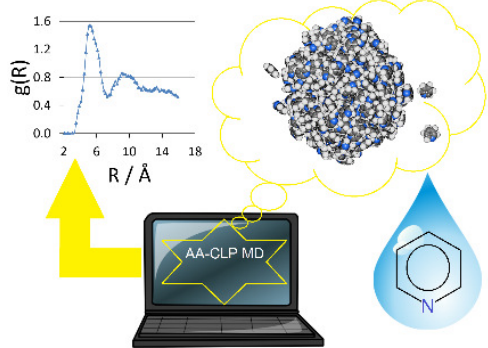

${ }_{15}$ Synopsis: In a few hours on a standard laptop, AA-CLP MD correctly reproduces thermodynamic properties of bulk liquids and provides information on the nanoscale dynamics of liquid nanoclusters. partial funding through the Unimi Development Plan-Line 2, Action B, project NOVAQ, nº PSR2015-1716FDEMA_08.

Conflict of interest. There are no conflicts to declare.

www.angelogavezzotti.it (2018); follow the CLPdyn link, file CLPmanual.doc.

${ }^{18}$ A. Gavezzotti and L. Lo Presti, Crystal Growth Des. 2015, 15, 3792-3803

${ }^{19}$ van Gunsteren, W. F., Billeter, S. R., Eising, A. A., Hunenberger, P. H., Kruger, P., Mark, A. E., Scott, W. R. P., Tironi, I. G. Biomolecular Simulation: The GROMOS96 Manual and User Guide, BIOMOS b.v., Zürich-Groningen, 1996.

${ }^{20}$ A. Gavezzotti, New. J. Chem. 2016, 40, 6848-6853.

${ }^{21}$ I. Cacelli, G. Cinacchi, G. Prampolini and A. Tani, J. Am. Chem. Soc. 2004, 126, 14278-14286

22 P. M. Piaggi and M. Parrinello, PNAS, 2018, 115, 10251-10256

${ }^{23}$ G. Gobbo, M. A. Bellucci, G. A. Tribello, G. Ciccotti, B. L. Trout, J. Chem. Theory Comput. 2018, 14, 959-972

${ }^{24}$ I. Gimondi and M. Salvalaglio, Mol. Syst. Des. Eng. 2018, 3, 243-252

${ }^{25}$ I. G. Tironi and W.F. van Gunsteren, Mol. Phys. 1994, 83, 381403.

${ }^{26}$ M. Haughney, M. Ferrario and R. MacDonald, J. Phys. Chem. 1987, 91, 4934-4940

${ }^{27}$ J. W. Handgraaf, T.S. van Erp and E. J. Meijer, Chem. Phys. Lett. 2003, 367, 617-624

${ }^{28}$ Z. Trumpakaj and B. Linde, J. Mol. Struct. 2015, 1085, 268-275.

${ }^{29}$ R. Destro, E. Sartirana, L. Loconte, R. Soave, P. Colombo, C. Destro and L. Lo Presti, Cryst. Growth Des. 2013, 13, 10, 4571-4582 